Revue d'histoire de l'Amérique française

DEV REVUE D.HISTOIRE DE L'AMÉRIQUE FRANÇAISE

\title{
Retour sur le projet de programme d'histoire " nationale " et son éducation à la citoyenneté
}

Félix Bouvier

Volume 61, numéro 2, automne 2007

URI : https://id.erudit.org/iderudit/018063ar

DOI : https://doi.org/10.7202/018063ar

Aller au sommaire du numéro

Éditeur(s)

Institut d'histoire de l'Amérique française

ISSN

0035-2357 (imprimé)

1492-1383 (numérique)

Découvrir la revue

Citer cet article

Bouvier, F. (2007). Retour sur le projet de programme d'histoire « nationale » et son éducation à la citoyenneté. Revue d'histoire de l'Amérique française, 61(2), 261-270. https://doi.org/10.7202/018063ar d'utilisation que vous pouvez consulter en ligne.

https://apropos.erudit.org/fr/usagers/politique-dutilisation/ 


\section{Retour sur le projet de programme d'histoire " nationale" et son éducation à la citoyenneté}

FÉLIX Bouvier

Didactique des sciences humaines

Département des sciences de l'éducation

Université du Québec à Trois-Rivières

\section{卫} el que nous y invite le Comité de rédaction de la Revue d'histoire de l'Amérique française, il ne nous est pas possible de demeurer silencieux et nous voulons donc répondre à l'article polémique de Michèle Dagenais et Christian Laville publié dans les pages de la revue au printemps $2007^{1}$. Ayant été impliqué dès les débuts ${ }^{2}$ dans le débat sur l'enseignement de l'histoire au deuxième cycle du secondaire québécois au printemps 2006 et ayant par la suite pris une grande part dans l'élaboration d'un dossier scientifique $^{3}$ sur ce thème, il nous semble devoir le faire.

D’entrée de jeu, il est intéressant de voir une historienne telle Michèle Dagenais défendre la logique didactique et historique ayant présidé à la confection du programme d'histoire et éducation à la citoyenneté au deuxième cycle du secondaire du ministère de l'Éducation, du Loisir et

1. Michèle Dagenais et Christian Laville, «Le naufrage du projet de programme d'histoire "nationale". Retour sur une occasion manquée accompagné de considérations sur l'éducation historique ", Revue d'histoire de l'Amérique française, 60,4 (printemps 2007): 517-550.

2. Félix Bouvier et Laurent Lamontagne, "Quand l'histoire se fait outil de propagande», Le Devoir, 28 avril 2006, A-9.

3. Félix Bouvier, dir., "Débat sur le programme d'enseignement de l'histoire au Québec», Bulletin d'histoire politique, 15,2 (hiver 2007): 4-106. 
du Sport ${ }^{4}$. En défendant deux projets de programme ${ }^{5}$ ayant précédé la version approuvée en novembre 2006 par le ministre Fournier, Madame Dagenais confirme à toutes fins utiles que toute règle doit avoir son exception. Elle est en effet la première à défendre l'esprit et l'essentiel des contenus relatifs à la question nationale de ces deux documents de travail, si on excepte les personnes consultées, ou encore les consultants, dans le processus menant à leur élaboration ${ }^{6}$.

\section{L'ESSENCE DU DÉBAT}

Michèle Dagenais et Christian Laville avancent que l'essentiel du débat repose sur «l'absence de dates charnières et d'événements d'un certain type, au profit de l'acquisition de compétences ${ }^{7} »$. Comme c'est souvent le cas dans cet article, il y a là du vrai et de l'interprétation problématique. Oui, l'absence de dates et d'événements d'un certain ordre - c'est-à-dire la trame, ou le récit historique priorisé - fut traitée avec indignation par nombre d'interlocuteurs. Pour ce qui est de l'approche par compétences, l'argumentation de nos deux auteurs laisse supposer que les principales critiques au projet de programme s'opposaient à la démarche par compétences, ou du moins la comprenaient fort mal. Or, la grande majorité des opposants à ce projet de programme ne remettaient pas en question l'approche par compétences, d'une part. D'autre part, une approche par compétences non seulement n'exclut pas les connaissances (sur le plan didactique, les compétences ont nécessairement besoin de connaissances pour fonctionner), mais elle peut en proposer un menu biaisé sur le plan historique ou chercher à en proposer une approche équilibrée. C’est précisément le biaisage constaté qui fut dénoncé avec autant de virulence

4. Dorénavant, nous utiliserons l'acronyme MELS pour désigner le ministère de l’Éducation, du Loisir et du Sport du gouvernement du Québec.

5. La première version du projet de programme, celle d'avril 2006, fut très vigoureusement dénoncée dans les médias. La seconde version (du 15 juin 2006) le fut aussi, tant et si bien que le ministre Jean-Marc Fournier força les concepteurs du programme à reculer une deuxième fois, après avoir élargi sensiblement semble-t-il la consultation à des historiens, des didacticiens et des organismes intéressées à l'histoire du Québec en plein été 2006. Nous fumes alors parmi les personnes consultées.

6. Le seul bémol connu et publié en ce qui touche l'orientation nationale promue de facto par ces deux projets est celui du didacticien Marc-André Éthier qui trouve la critique virulente à ce chapitre "erronée». "Apprendre à exercer sa citoyenneté à l'aide de l'histoire», Bulletin d'histoire politique, 15,2 (hiver 2007): 53. Éthier se veut par ailleurs critique sous d'autres aspects davantage sociaux quant aux orientations du programme.

7. M. Dagenais et C. Laville, «Le naufrage du projet de programme d'histoire "nationale"...», op. cit., 520 . 
entre avril et septembre 2006 et, malgré les nuances, une convergence confinant presque à l'unanimité.

Pour ces deux auteurs, le projet de programme a été accusé d'occulter le "nous» des "anciens Canadiens français, [...] sans fondement documentés ${ }^{8} »$. En fait, il y a tellement de documentation à ce sujet (des dizaines d'articles y réfèrent) que nous nous bornerons à tracer et rappeler ici quelques grands traits (allant de 1608 à 1848) du seul projet de programme d'histoire et éducation à la citoyenneté si dénoncé d’avril 2006. Les Canadiens devenus Canadiens français, puis Québécois autour de 1960 se définissaient eux-mêmes (et l'étaient aussi par les Anglo-Britanniques,

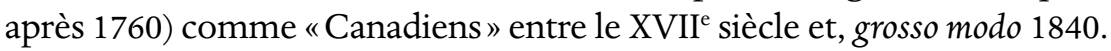
Le projet de programme ne faisait pas cette nuance lorsqu'il abordait la Nouvelle-France... Il traitait des "Canadiens» d'alors en ne mentionnant pas que la majorité des "Canadiens » d'aujourd'hui sont d'abord et avant tout anglophones et vivent très majoritairement à l'extérieur du Québec. Tout le reste du projet de programme était à l'avenant.

L’Acte de Québec de 1774 était passé sous silence, lui qui explique entre autres le particularisme juridique du Québec encore aujourd'hui dans l'ensemble canadien par un code civil d'origine napoléonien et français. La période postconquête de 1760-1848 était présentée comme «L’accession à la démocratie sous le régime britannique», ce qui constituait une réalité sociale, une subdivision fort importante sur le plan didactique, conceptuel et pédagogique. Le contenu de cette réalité sociale était en corrélation avec son titre, c'est-à-dire qu'il mettait en vedette le libéralisme britannique $^{9}$. Ce n'est pas faux de présenter les choses ainsi historiquement. C'est toutefois fortement orienté, surtout lorsque la finalité du processus s'adresse à des adolescents qui n'ont pas d'armature historique pour nuancer un programme et qui, au surplus, seront éventuellement évalués en lien avec ledit programme... L'autre côté de la médaille, c'est que la période 1760-1848 en est une de tentatives d'assimilation incessantes de la minorité anglo-britannique envers la majorité canadienne (française) de la vallée du Saint-Laurent, comprenant sa mise en minorité définitive politiquement par l'Acte d'Union en 1840.

Nos deux auteurs oublient commodément qu'en taisant le plus systématiquement possible des moments positifs et les moments conflictuels de la conscience nationale de la grande majorité des Québécois contemporains

8. Ibid., 522.

9. Le libéralisme britannique est la base idéologique même du fédéralisme canadien. 
à travers leur histoire, tout en niant à travers un programme d'enseignement jusqu'à l'existence de cette conscience le plus possible, ils favorisent objectivement les fondements mêmes d'un nationalisme fédéraliste canadian inclusif.

Les historiens doivent sans doute éviter de faire la promotion d'une quelconque idéologie politique particulière. Ils doivent notamment éviter de faire dans leur classe la promotion du fédéralisme canadien ou de la souveraineté du Québec. Mais doivent-ils en plus éviter de faire toute référence à la nation québécoise ou à la nation canadienne-française, à l'histoire nationale et à la question nationale pour éviter de se compromettre politiquement? L'évacuation de toute référence à la nation québécoise n'est-elle pas, bien au contraire, une position qui compromet les historiens qui la proposent ${ }^{10}$ ?

Ainsi donc, c'est par dizaines que des historiens et intellectuels québécois dénoncent le fait que la trame historique priorisée dans le projet de programme du MELS, et l'édulcoration constatée quant à la question nationale, renvoient en fait à un récit ne permettant que bien peu d'approcher cette question objectivement. C'est ce constat qui est grave en ce qui touche l'intégrité du présent et du passé québécois. Ce l'est toutefois encore davantage pour l'avenir. Car, comme le dit un important didacticien, le grand rôle de l'histoire consiste à préparer le futur. «La fonction de la mémoire, il faut le redire, n’est pas de célébrer la messe du passé, c'est d'aider à imaginer l'avenir ${ }^{11}$.»

\section{HISTOIRE, ÉDUCATION CIVIQUE ET ÉDUCATION À LA CITOYENNETÉ}

Laville et Dagenais développent ensuite une théorie contestable, mais qui est centrale pour comprendre leur thèse. Du récit fondateur en ce qui touche la nation enseignée en histoire dans la première moitié $\mathrm{du} \mathrm{xx}^{\mathrm{e}}$ siècle au Québec (et dans le reste de l'Occident, devine-t-on), ils nous disent qu'il s'agit là d'éducation civique. Cette éducation civique est perçue comme rétrograde et elle est surtout associée à un "récit fermé ${ }^{12}$ », récit auquel sont assimilés les opposants aux deux projets de programme un peu partout dans le développement de l'article. Inversement, la logique didactique soutenant ces projets de programme mènerait à un récit ouvert permet-

10. «Le programme d'histoire au secondaire. Une nouvelle version à recentrer", Le Devoir, 28 septembre 2006, A-7 (lettre signée par vingt-trois intellectuels québécois, dont une majorité d'historiens).

11. Henri Moniot, Didactique de l'histoire (Paris, Nathan, 1993), 158.

12. M. Dagenais et C. Laville, «Le naufrage du projet de programme d'histoire "nationale”..., op. cit., 536. 
tant l'élaboration de savoirs pour que l'élève puisse construire une compréhension du social. Cela renverrait à une mutation progressive de l'éducation civique vers l'éducation à la citoyenneté. Les aspects de l'histoire permettant une compréhension des origines nationales canadiennesfrançaises de l'ensemble des Québécois actuels n'auraient plus leur place étant donné les prérogatives modernes de l'éducation à la citoyenneté. Comment interpréter autrement ce qu'ils nous en disent? «Progressivement, l'enseignement de l'histoire à l'école complète son passage d'une éducation civique nationale à l'éducation à la citoyenneté ${ }^{13}$."

\section{ÉDUCATION À LA CITOYENNETÉ, HISTOIRE ET QUESTION NATIONALE}

Évidemment, Dagenais et Laville n'ont pas d'appuis didactiques cités pour soutenir la prise de position énoncée ci-dessus et ce qu'elle sous-tend, car cela ne tient pas la route. Nier ou omettre dans un projet de programme une quantité de faits historiques dont l'importance est profonde dans la construction de l'identitaire national québécois n'est-ce pas une posture qui renvoie directement à un récit fermé sur ce thème? Poser la question, c'est y répondre à notre avis. Ainsi, à titre d'exemples sur l'histoire du Québec au $\mathrm{xx}^{\mathrm{e}}$ siècle, comment de jeunes Québécois peuvent-ils avoir les éléments nécessaires pour comprendre une partie appréciable du monde actuel si un projet de programme omet complètement toute la question du néo-nationalisme québécois des années 1950 à 1980 et ses phénomènes convergents? Ou encore, comment est-ce possible de comprendre l'évolution du Québec depuis une génération si les trois référendums de 1980, 1992 et 1995 sont passés sous silence et qu'en plus on met en lumière pour l'élève et l'enseignant «l'enchâssement de la Charte canadienne des droits et libertés dans la Loi constitutionnelle ${ }^{14}$ » de 1982, sans même mentionner que le Québec n’a pas signé cette constitution?

Selon la logique de Michèle Dagenais et Christian Laville, il s'agit là de la version qu'il faut surtout regretter. Dans ce contexte, comment leur est-il possible de défendre le fait que le développement de l'esprit critique des élèves est censé être un jalon incontournable sur le plan didactique de l'apprentissage en histoire? Au sujet de la double question nationale canadienne, Marie McAndrews vise particulièrement juste lorsqu'il est question des adolescents, ce que les praticiens québécois en histoire nationale au secondaire savent fort bien d'ailleurs.

13. Ibid., 537.

14. À propos de certains des aspects majeurs de cette occultation historique, voir Félix Bouvier et Laurent Lamontagne, "Quand l'histoire se fait outil de propagande», op. cit. 
Dans les sociétés où deux instances étatiques se disputent l'allégeance des citoyens, l'éducation à la citoyenneté, à défaut de prendre clairement position dans ce débat, ce qui n'est évidemment pas le rôle de l'école, peut-elle véritablement faire l'économie d'un débat sur cette question sans risquer de tomber dans l'insignifiance aux yeux des élèves, notamment au secondaire ${ }^{15}$ ?

Au nom de l'immigration diversifiée et accentuée (mais non chiffrée) des dernières décennies, nous disent Dagenais et Laville, il faut faciliter l'intégration de ces nouveaux arrivants par le relais de l'histoire et l'éducation à la citoyenneté. En favorisant en ce nom une approche taisant des pans fort importants de l'histoire nationale, non seulement on ne favorise pas l'intégration des immigrants, mais on contribue à l'érection d'un mur d'incompréhension sur la question fondamentale de l'identité nationale entre ces nouveaux arrivants ou les enfants d'immigrants d'un côté et ce que l'on nomme les Québécois de souche de l'autre. Ces derniers en effet, par leur culture première et familiale, ce qui inclut la mémoire ancestrale qui leur est transmise, ont bien davantage d'outils pour comprendre ce que ces deux projets de programme tentaient de leur cacher sur le plan de l'histoire de ce qui est à notre avis l'indéniable nation québécoise. Voyons ce qu'en dit Bernard Delmotte dans son article «La citoyenneté des résidents étrangers » : "Notion évolutive et n'ayant pas la même signification d'un pays à l'autre, le concept de citoyenneté est le résultat d'une histoire nationale : la citoyenneté à la française issue de la Révolution ne veut pas dire la même chose que la "citizenship" britannique influencée par l'épopée du Commonwealth ${ }^{16}$.»

\section{HISTOIRE DU QUÉBEC ET SON ENSEIGNEMENT}

Après avoir référé à des apprentissages de l'histoire au Québec de la première moitié du $\mathrm{xx}^{\mathrm{e}}$ siècle qui ne sont en effet plus d'actualité, Dagenais et Laville se servent de cet exemple pour justifier une approche didactique en elle-même fort probante. Pour pallier «l'éventuelle obsolescence» des apprentissages, nous disent-ils, c'est la méthode historique et ses concepts qui doivent avoir le haut du pavé ${ }^{17}$.

15. Marie McAndrew, «Éducation interculturelle et éducation à la citoyenneté dans les nouveaux programmes québécois : une analyse critique», dans Fernand Ouellet, dir., Quelle formation pour l'éducation à la citoyenneté? (Québec, Les Presses de l'Université Laval, 2004), 32-33.

16. Bernard Delmotte, "La citoyenneté des résidents étrangers», dans Claude Carpentier, Identité nationale et enseignement de l'histoire. Contextes européens et africains (Paris/Montréal, L'Harmattan, 1999), 169.

17. Les auteurs mentionnent «Le sacrifice héroïque» de Dollard des Ormaux et le développement d'un certain «esprit social chrétien», 541. 
Nous sommes bien d'accord avec cette approche didactique basée sur la méthode et la pensée historique par le relais de concepts appropriés. Encore faut-il cependant que ces derniers soient véritablement appropriés et que leur choix soit fait de façon ouverte ${ }^{18}$, d'une part. D'autre part, la démarche par compétences sur laquelle cela repose est toujours bien présente dans le programme signé par le ministre et que Dagenais et Laville associent à un échec. Nous croyons que ce ne sont pas les dates rajoutées en novembre 2006 qui posent véritablement problème aux deux polémistes puisque cet ajout de dates était déjà bien présent dans la deuxième version du projet de programme, celle du 15 juin 2006. À ce moment, «le didacticien Christian Laville, de l’Université Laval, n'avait que de bons mots pour le document: ils ont réussi à tenir compte des critiques de façon exemplaire, de toutes les critiques ${ }^{19}$ ". Alors que s'est-il passé entre juin et novembre 2006 pour que Monsieur Laville et Madame Dagenais parlent de "naufrage »? Tout simplement, la réponse est à notre avis due au fait que le thème de la nation n'est plus nié par le relais de certains concepts priorisés par le programme dorénavant officiel ${ }^{20}$.

Pour notre part, nous croyons que le problème du programme (et, évidemment, encore davantage de ses versions antérieures) se pose exactement à l'inverse. Les ajouts conceptuels relatifs au thème de la nation canadienne, canadienne-française, puis québécoise (et sa double réalité nationale depuis 1840) ne sont, dans la version actuelle, que du rapiéçage. L'esprit cherchant à les nier ou à en minimiser l'importance a toujours préséance et c'est l'approche qu'il faudra éventuellement modifier. L'essence même du débat est là. Paul Inchauspé a bien relevé ce silence sur cette question qui date du rapport Lacoursière: "Dans ce contexte, toute histoire nationale (nation ethnique? nation civique?), est rapidement considérée comme histoire nationaliste et par crainte d'une telle accusation, ou on se tait et on fait de l'histoire nationale sans le dire et avec mauvaise conscience ${ }^{21}$.»

Alors, oui l'élève doit pouvoir développer, par le relais de compétences propres à l'histoire, une démarche intellectuelle qui favorisera des habi-

18. Semble-t-il que neuf des onze historiens consultés par le MELS pour la confection du projet de programme sont affiliés à l'Université Laval, ce que la documentation officielle du MELS ne révèle toutefois pas, sauf erreur.

19. Antoine Robitaille, «L'Histoire retrouvera toutes ses dates», Le Devoir, 16 juin 2006, A-1.

20. MELS, Programme de formation de l'école québécoise, histoire et éducation à la citoyenneté du deuxième cycle du secondaire (Gouvernement du Québec, 2006), 105 p.

21. Paul Inchauspé, «La place de l'histoire et des perspectives historiques dans la réforme du programme d'études», texte inédit présenté à l'occasion du congrès annuel de la Société des professeurs d'histoire du Québec, 9, Laval, 26 octobre 2007. 
letés cognitives ${ }^{22}$, assurant à la fois une meilleure rétention des apprentissages effectués et, surtout, des possibilités nettement supérieures de transférer dans sa vie courante et dans sa vie adulte les habiletés historicointellectuelles ainsi sollicitées.

Toutefois, dans le contexte d'un cours d'histoire du Québec-Canada, il ne saurait être question que cette approche exclut ce qui touche la question nationale, dans un programme d'enseignement national de surcroît. En fait, avoir des outils et des clés explicatives pertinentes est essentiel à la compréhension du contexte historique national où vivent et où vivront les adolescents à qui cet enseignement est destiné. Voyons quelques réflexions du Rapport Inchauspé à ce sujet:

[...] parce qu'il n'y a pas d'intégration réussie dans une société sans qu'à l'école on aide à s'approprier, par l'enseignement, les traditions culturelles de cette société. De ce point de vue, la situation de l'enfant né au Québec est la même que celle de l'immigrant nouvellement arrivé. [...] Ne pas connaître son passé, c'est s'exposer à le reproduire. [...] Dans un monde depuis longtemps interdépendant, ignorer sa propre histoire, c'est souvent se condamner à ignorer l'histoire des autres ${ }^{23}$.

Nier ou couvrir insuffisamment, ou encore de façon erronée, l'histoire nationale ne peuvent que favoriser l'incompréhension de la réalité nationale dans laquelle les adolescents évoluent et évolueront et ne favorisent nullement le vivre ensemble souhaité ${ }^{24}$. Et la nation à laquelle adhère de façon prioritaire une grande majorité de Québécois, toutes tendances politiques confondues, est celle du Québec, que l'on aime ça ou non. Évidemment, l'espace national canadien n'a pas à être occulté pour autant, en tout respect avec l'histoire du Québec-Canada et son présent.

On a aussi passablement reproché aux projets de programme et au programme officiel de négliger l'aspect politique, entre autres, en n'incluant pas en quatrième secondaire une réalité sociale distincte basée sur l'histoire politique ${ }^{25}$, alors que les autres aspects (économique, social et culturel) fondamentaux à la vie en société ont droit à une telle couverture didactique et pédagogique. Telle n'est manifestement pas la position que

22. Voir à ce sujet, entre autres, Robert Martineau, L'histoire à l'école, matière à penser (Paris/ Montréal, L’Harmattan, 1999), 400 p.

23. Rapport du Groupe de travail sur la réforme du curriculum, Réaffirmer l'école (Gouvernement du Québec, 1997), 35-36.

24. À ce sujet, voir aussi Félix Bouvier, «Le multiculturalisme, l’éducation à la citoyenneté et l'enseignement de l'histoire au Québec», Traces, 45,2 (mars-avril 2007): 12-15.

25. Idem. 
veulent étayer Madame Dagenais et Monsieur Laville. Pour eux, «l’histoire scolaire conserve des perspectives nationales et une chronologie générale à base politique qui n'est plus le fait des historiens ${ }^{26}{ }^{\prime}$. Au sujet des historiens, nos deux auteurs soulignent avec justesse qu'ils se sont quelque peu éloignés des réalités propres à l'enseignement au secondaire depuis quelques années. Là où c'est plus problématique, c'est lorsque Laville et Dagenais s'interrogent quant à la prise de position évoquée $e^{27}$ en septembre 2006 de plus d'une quinzaine d'entre eux ${ }^{28}$, ils questionnent rien de moins que leur objectivité. «D’ailleurs, parlent-ils en historiens ou en militants ${ }^{29}$ ?» Cette prémisse plus que questionnable est ensuite appuyée de la note suivante: "C'est un fait que signale le didacticien Félix Bouvier $^{30}$. " Cette référence est une "incompréhension" à relever et ce n'est absolument pas ce qui est libellé dans ce que Laville et Dagenais évoquent. Plus important pour les fondements du débat en cours, il y est plutôt écrit ceci: «Michel Seymour pourfend la pensée de Jocelyn Létourneau [historien de l'Université Laval] pour qui les références à la nation québécoise doivent être évitées chez ses intellectuels alors que lui-même fait la promotion acharnée de notre "canadianité" ${ }^{1}$ ".

\section{CONCLUSION}

Mémoire nationale et démocratie n'ont pas à s'exclure, elles doivent se compléter, surtout dans l'enseignement de l'histoire. À notre connaissance d'ailleurs, jamais personne n'a dit autre chose au Québec, hormis évidemment les gens qui accusent impunément de nationalisme ethnique ceux qui seraient nationalistes. Ne serait-ce pas plutôt l'inverse qui est en cause ici? Au cours de leur histoire, les Québécois n’ont-ils pas été bien davan-

26. M. Dagenais et C. Laville, «Le naufrage du projet de programme d'histoire "nationale”..., op. cit, 544.

27. "Le programme d'histoire au secondaire. Une nouvelle version à recentrer», op. cit.

28. Parmi ces historiens, on retrouve, entre autres, Denise Angers, Éric Bédard, Jean-Paul Bernard, Robert Comeau, Micheline Dumont (aussi didacticienne émérite), Jean-Marie Fecteau, Lucia Ferretti, Yves Gingras, Jacques Lacoursière, Yvan Lamonde, Martin Pâquet, Jacques Rouillard, Michel Sara-Bournet et Denis Vaugeois. En nous limitant ici aux historiens, nous avons oublié des éléments signataires importants, tels Jacques Beauchemin, Alain-G. Gagnon, Laurent Lamontagne, Michel Seymour, Guy Rocher et autres universitaires.

29. M. Dagenais et C. Laville, «Le naufrage du projet de programme d'histoire "nationale”..., op. cit, 545.

30. Dagenais et Laville réfèrent ici à Félix Bouvier, «Présentation du dossier: Débat sur l'enseignement de l'histoire nationale au secondaire", Bulletin d'histoire politique, 15,2 (hiver 2007): $7-8$.

31. Ibid., 7. 
tage victimes de racisme qu'ils en ont eux-mêmes fait subir ${ }^{32}$ ? Bien sûr, cela ne justifierait en aucun cas de promouvoir de quelque façon que ce soit l'inverse, mais la culpabilité injustifiée a ses limites. L'enseignement de l'histoire nationale du Québec-Canada n'a pas à en faire les frais.

Il faudrait donc que ce programme d'enseignement soit revu et associe bien davantage nation et démocratie. Pour terminer, laissons intervenir le didacticien Christian Laville qui ne pourra nous semble-t-il qu'être d'accord avec ce qui suit, étant donné la désolation (le fameux "naufrage») que constitue pour lui le programme. «Examinant cinq programmes sur vingt-cinq ans, l'auteur y montre que seuls s'implantent vraiment dans les pratiques scolaires les programmes qui recueillent suffisamment l'agrément des enseignants, quels que soient leur forme, leurs objectifs, leur contenu ou l'autorité dont ils proviennent ${ }^{33}{ }^{\prime}$. Tel que l'énonce un certain article $^{34}$, il est bien possible en effet que ce soit les enseignants québécois qui aient le dernier mot. Il serait bien étonnant toutefois qu'ils rejettent ce programme pour les mêmes raisons que Michèle Dagenais et Christian Laville. Il s'agit donc d'une histoire à suivre.

32. Voir à ce sujet: Normand Lester, Le livre noir du Canada anglais (Montréal, Les intouchables, 2001 et 2002), tome I et II.

33. Christian Laville, «La recherche empirique en éducation historique, mise en perspective et orientations actuelles", dans Perspectives documentaires en éducation (Paris, INRP, 2001), 75.

34. Sophie Doucet, "Je me souviens de quoi? La réforme de l'enseignement de l'histoire pourrait marquer la fin d'une certaine idée du "nous" québécois », L'Actualité, $1{ }^{\text {er }}$ septembre 2007, 21-25. 